\title{
Polynilpotent Capability of Finitely Generated Abelian Groups
}

\author{
Mashayekhy Behrooz $^{1, *}$, Parvizi Mohsen ${ }^{1}$, and Kayvanfar Saeed ${ }^{1,2}$ \\ ${ }^{1}$ Department of Pure Mathematics, Center of Excellence in Analysis on Al- \\ gebraic Structures, Ferdowsi University of Mashhad, Mashhad, Iran. \\ ${ }^{2}$ Institute for Studies in Theoretical Physics and Mathematics, Tehran, Iran.
}

\begin{abstract}
In this paper we determine all finitely generated abelian groups which are varietal capable with respect to the variety of polynilpotent groups. This result is a vast generalization of the famous Baer's result about capability of finitely generated abelian groups.
\end{abstract}

Keywords: Varietal capability; Finitely generated Abelian groups; Polynilpotent variety; Baer invariant.

AMS subject classifications: 20E34, 20E10, 20K01.

\section{Introduction}

In 1938 Baer [1] investigated the conditions for a group to be the inner automorphism group of another group. Hall and Senior [3] called such groups capable. P. Hall in his landmark paper [4], noted that characterization of capable groups is important in classifying groups of prime power order. Baer [1] succeeded to determine all capable groups among the direct sums of cyclic groups, and hence finitely generated abelian groups. The third author in his joint paper in 1997 [6], generalized the notion of capability to any variety of groups which was called $\mathcal{V}$-capability.

The aim of this work is determining all finitely generated abelian groups which are varietal capable with respect to the variety of polynilpotent groups of class row $\left(c_{1}, c_{2}, \ldots, c_{t}\right)$, that is $\mathcal{N}_{c_{1}, c_{2}, \ldots, c_{t}}$. This result is a vast generalization of Baer's work in the case of finitely generated abelian groups.

\footnotetext{
${ }^{*}$ Correspondence to: Mashayekhy Behrooz, Department of Pure Mathematics, Center of Excellence in Analysis on Algebraic Structures, Ferdowsi University of Mashhad, P.O.Box 1159-91775, Mashhad, Iran. Email: mashf@math.um.ac.ir

${ }^{*}$ Received: 3 october 2009

http://www.i-asr.org 


\section{Preliminaries}

We shall assume that the reader is familiar with the notion of variety of groups and the verbal and the marginal subgroups. The notion of Baer invariant is also considered to be known (see [6]). The following definition is of several usage in this article.

Definition 2.1. Let $\mathcal{V}$ be any variety of groups defined by a set of laws $V$, and $G$ be any group. $G$ is called $\mathcal{V}$-capable if there exists a group $E$ which satisfies $G \cong E / V^{*}(E)$, where $V^{*}(E)$ is the marginal subgroup of $E$ with respect to $\mathcal{V}$.

It should be noted that although two different sets of words can generate a variety but the marginal subgroups with respect to these sets of words coincide, so the terminology $\mathcal{V}$-capability has no ambiguity. According to the above definition capable groups are $\mathcal{A}$-capable, where $\mathcal{A}$ is the variety of abelian groups.

The following definition and theorem are found in [6] and contains a necessary and sufficient condition for a group to be $\mathcal{V}$-capable.

Definition 2.2. Let $\mathcal{V}$ be any variety and $G$ be any group. Then $V^{* *}(G)$ is defined as follows.

$$
V^{* *}(G)=\cap\left\{\psi\left(V^{*}(E)\right) \mid \psi: E \stackrel{\text { onto }}{\longrightarrow} G, \operatorname{ker} \psi \subseteq V^{*}(E)\right\} .
$$

Theorem 2.3. With the above notations and assumptions $G / V^{* *}(G)$ is the largest quotient of $G$ which is $\mathcal{V}$-capable, and hence $G$ is $\mathcal{V}$-capable if and only if $V^{* *}(G)=1$.

The following theorem and its consequences state relationship between $\mathcal{V}$-capability and Baer invariants.

Theorem 2.4. ( $[6]$ ). Let $\mathcal{V}$ be any variety of groups, $G$ be any group, and $N$ be a normal subgroup of $G$ contained in the marginal subgroup $V^{*}(G)$. Then the natural homomorphism $\mathcal{V} M(G) \longrightarrow \mathcal{V} M(G / N)$ is injective if and only if $N \subseteq V^{* *}(G)$, where $\mathcal{V} M(G)$ is the Baer invariant of $G$ with respect to $\mathcal{V}$.

In finite case the following corollary is useful to be applied.

Corollary 2.5. ( $[7])$. Let $\mathcal{V}$ be any variety and $G$ be any group with $V(G)=1$. Assume that $\mathcal{V} M(G)$ and $\mathcal{V} M(G / N)$ are finite groups for a normal subgroup $N$ of $G$, then the natural homomorphism $\mathcal{V} M(G) \longrightarrow \mathcal{V} M(G / N)$ is injective if and only if $|\mathcal{V} M(G / N)|=|\mathcal{V} M(G)|$.

The following result is a consequence of Theorem 2.4.

Corollary 2.6. An abelian group $G$ is $\mathcal{V}$-capable if and only if the natural homomorphism $\mathcal{V} M(G) \longrightarrow \mathcal{V} M(G /<x>)$ has a non-trivial kernel for all non-identity elements $x$ in $G$.

In order to use Theorem 2.4 we need the explicit structure of the Baer invariants of finitely generated abelian groups with respect to the variety of polynilpotent groups. The following give us the structure. 
Theorem 2.7. ( $[5])$. Let $\mathcal{N}_{c_{1}, c_{2}, \ldots, c_{t}}$ be the polynilpotent variety of class row $\left(c_{1}, c_{2}, \ldots, c_{t}\right)$ and $G \cong \mathbb{Z}^{(m)} \oplus \mathbb{Z}_{n_{1}} \oplus \ldots \oplus \mathbb{Z}_{n_{k}}$ be a finitely generated abelian group, where $n_{i+1} \mid n_{i}$ for all $1 \leq i \leq k-1$. Then an explicit structure of the polynilpotent multiplier of $G$ is as follows.

$$
\mathcal{N}_{c_{1}, c_{2}, \ldots, c_{t}} M(G) \cong \mathbb{Z}^{\left(f_{m}\right)} \oplus \mathbb{Z}_{n_{1}}^{\left(f_{m+1}-f_{m}\right)} \oplus \ldots \oplus \mathbb{Z}_{n_{k}}^{\left(f_{m+k}-f_{m+k-1}\right)}
$$

where $f_{i}=\chi_{c_{t}+1}\left(\chi_{c_{t-1}+1}\left(\ldots\left(\chi_{c_{1}+1}(i)\right) \ldots\right)\right)$ for all $m \leq i \leq m+k$.

To use Theorem 2.4 and Corollary 2.5, we need the structure of subgroups of finitely generated abelian groups as follows.

Theorem 2.8. ( $[7])$. Let $G \cong \mathbb{Z}^{(m)} \oplus \mathbb{Z}_{n_{1}} \oplus \ldots \oplus \mathbb{Z}_{n_{k}}$ be a finitely generated abelian group, where $n_{i+1} \mid n_{i}$ for all $1 \leq i \leq k-1$ and $H \leq G$ be a finite subgroup of $G$. Then $H \cong \mathbb{Z}_{m_{1}} \oplus \ldots \oplus \mathbb{Z}_{m_{k}}$, where $m_{i+1} \mid m_{i}$ for all $1 \leq i \leq k-1$ and $m_{i} \mid n_{i}$ for all $1 \leq i \leq k$.

\section{Main Results}

First of all we state two theorems of Baer, Burns and Ellis, which determine all $\mathcal{N}_{c^{-}}$ capable finitely generated abelian groups, in which $\mathcal{N}_{c}$ is the variety of nilpotent groups of class at most $c$. The case $c=1$ is permissible and refers to capability.

The following theorem is not just what Baer proved, but a conclusion of it as a special case.

Theorem 3.1. (R. Baer [1]). Let $G \cong \mathbb{Z}^{(m)} \oplus \mathbb{Z}_{n_{1}} \oplus \ldots \oplus \mathbb{Z}_{n_{k}}$ be a finitely generated abelian group, where $n_{i+1} \mid n_{i}$ for all $1 \leq i \leq k-1$. Then $G$ is capable precisely when

(i) $m \geq 2$; or

(ii) $m=0, k \geq 2$ and $n_{1}=n_{2}$.

Theorem 3.2. (J. Burns and G. Ellis [2]). A finitely generated abelian group is $\mathcal{N}_{c^{-}}$ capable if and only if it is capable.

According to the above theorem, the condition for a finitely generated abelian group to be $\mathcal{N}_{c}$-capable is just the condition for capability of it. It is of interest to know which varieties behave like as nilpotent varieties in case of capability of finitely generated abelian groups. Answering this question in general case need much more information, but in case of some outer commutator varieties, more precisely polynilpotent varieties, we intend to answer it. In the rest we see that not all polynilpotent varieties behave like as nilpotent varieties in case of capability, but a large class of them do. To show varieties which not behave so we give the following example.

Example 3.1. Let $G \cong \mathbb{Z}_{n} \oplus \mathbb{Z}_{n}$, where $n \in \mathbb{N}$. One may easily show that $G$ and all its quotients are at most two-generator abelian groups, so by Theorem $2.7 \mathcal{S}_{2} M(G / N)=0$ for any normal subgroup of $G, N$ say, in which $\mathcal{S}_{2}$ is the variety of metabelian groups. Now Theorem 2.4 implies $G$ is not $\mathcal{S}_{2}$-capable. 
Now the main goal of this article is presented, which is classifying all $\mathcal{N}_{c_{1}, c_{2}, \ldots, c_{t}}{ }^{-}$ capable groups in the class of finitely generated abelian groups. According to Theorem 2.7 if $t \geq 2$ and $c_{1}=1$, then $\mathcal{N}_{c_{1}, c_{2}, \ldots, c_{t}} M(G)=0$ whenever $G$ has at most two generators, but if $t=1$ or $c_{1} \geq 2$, then $\mathcal{N}_{c_{1}, c_{2}, \ldots, c_{t}} M(G)=0$ only when $G$ is cyclic. Hence we need to separate these two cases, and the rest of work shows the results are different.

We only state the proof in the case $t \geq 2$ and $c_{1}=1$, the proof of the other case is similar. Just for the sake of clarity we separate the procedure in two cases; finite and infinite.

Case one: Finite case.

Throughout the following two lemmas we assume that $t \geq 2$ and $c_{1}=1$.

Lemma 3.3. Let $G \cong \mathbb{Z}_{n_{1}} \oplus \ldots \oplus \mathbb{Z}_{n_{k}}$ be a finite abelian group, where $n_{i+1} \mid n_{i}$ for all $1 \leq i \leq k-1$. If $G$ is $\mathcal{N}_{c_{1}, c_{2}, \ldots, c_{t}}$-capable then $k \geq 3$ and $n_{1}=n_{2}=n_{3}$.

Proof. By contrary assume we have $k \leq 2$ or not $n_{1}=n_{2}=n_{3}$. If $k \leq 2$ then similar to Example $3.1 G$ and all its quotients are at most two-generator abelian groups so by Theorem $2.7 \mathcal{N}_{c_{1}, c_{2}, \ldots, c_{t}} M(G / N)=0$ for any normal subgroup $N$ of $G$, so by Theorem $2.4 G$ is not $\mathcal{N}_{c_{1}, c_{2}, \ldots, c_{t}}$-capable. On the other hand if, for example, $n_{1} \neq n_{2}$, we introduce a normal subgroup of $G, N$ say; for which the natural homomorphism $\mathcal{N}_{c_{1}, c_{2}, \ldots, c_{t}} M(G) \longrightarrow \mathcal{N}_{c_{1}, c_{2}, \ldots, c_{t}} M(G / N)$ is injective. Put $N$ the subgroup generated by $\left(\bar{n}_{2}, \overline{0}, \ldots, \overline{0}\right)$. It is easy to see that $N \cong \mathbb{Z}_{\frac{n_{1}}{n_{2}}}$ and $G / N \cong \mathbb{Z}_{n_{2}} \oplus \mathbb{Z}_{n_{2}} \ldots \oplus \mathbb{Z}_{n_{k}}$. Now Theorem 2.7 shows that $\left|\mathcal{N}_{c_{1}, c_{2}, \ldots, c_{t}} M(G)\right|=\left|\mathcal{N}_{c_{1}, c_{2}, \ldots, c_{t}} M(G / N)\right|$, hence by Corollary 2.5 we have the injectivity of $\mathcal{N}_{c_{1}, c_{2}, \ldots, c_{t}} M(G) \longrightarrow \mathcal{N}_{c_{1}, c_{2}, \ldots, c_{t}} M(G / N)$. So the result holds by Theorem 2.4. The second case for which we have $n_{1}=n_{2} \neq n_{3}$ has a similar proof by putting $N=<\left(\overline{0}, \bar{n}_{3}, \overline{0}, \ldots, \overline{0}\right)>$.

The above lemma presents a necessary condition for a finite abelian group to be $\mathcal{N}_{c_{1}, c_{2}, \ldots, c_{t}}$-capable. The following shows it is sufficient.

Lemma 3.4. Let $G \cong \mathbb{Z}_{n_{1}} \oplus \ldots \oplus \mathbb{Z}_{n_{k}}$ be a finite abelian group, where $n_{i+1} \mid n_{i}$ for all $1 \leq i \leq k-1$ in which $k \geq 3$ and $n_{1}=n_{2}=n_{3}$. Then $G$ is $\mathcal{N}_{c_{1}, c_{2}, \ldots, c_{t}}$-capable.

Proof. By Theorem 2.4 it is enough to show that for an arbitrary normal subgroup $N$ of $G, \mathcal{N}_{c_{1}, c_{2}, \ldots, c_{t}} M(G) \longrightarrow \mathcal{N}_{c_{1}, c_{2}, \ldots, c_{t}} M(G / N)$ is not injective. In finite abelian groups each quotient is isomorphic to a subgroup and vice versa. Now let $N$ be an arbitrary normal subgroup of $G$, then $G / N$ is isomorphic to a subgroup of $G, H$ say; so by Theorem $2.8 H \cong \mathbb{Z}_{m_{1}} \oplus \ldots \oplus \mathbb{Z}_{m_{k}}$, where $m_{i+1} \mid m_{i}$ for all $1 \leq i \leq k-1$ and $m_{i} \mid n_{i}$ for all $1 \leq i \leq k$. Computing $\mathcal{N}_{c_{1}, c_{2}, \ldots, c_{t}} M(G)$ and $\mathcal{N}_{c_{1}, c_{2}, \ldots, c_{t}} M(H)$ using Theorem 2.7, shows that $\left|\mathcal{N}_{c_{1}, c_{2}, \ldots, c_{t}} M(G)\right|=\left|\mathcal{N}_{c_{1}, c_{2}, \ldots, c_{t}} M(H)\right|$ if and only if $m_{i}=n_{i}$ for all $3 \leq i \leq k$, but $n_{1}=n_{2}=n_{3}$ by hypothesis which implies $n_{1}=m_{1}$ and $n_{2}=m_{2}$ which is equivalent to $H=G$ or $N=0$. Now by Theorem $2.4 G$ is $\mathcal{N}_{c_{1}, c_{2}, \ldots, c_{t}}$-capable.

Case two: Infinite case.

Similar to the previous case, through the following two lemmas we assume that $t \geq 2$ and $c_{1}=1$. 
Lemma 3.5. Let $G \cong \mathbb{Z}^{(m)} \oplus \mathbb{Z}_{n_{1}} \oplus \ldots \oplus \mathbb{Z}_{n_{k}}$ be an infinite finitely generated abelian group, where $n_{i+1} \mid n_{i}$ for all $1 \leq i \leq k-1$. If $G$ is $\mathcal{N}_{c_{1}, c_{2}, \ldots, c_{t}}$-capable then $m \geq 3$.

Proof. We show that if $m \leq 2$, then there exists a normal subgroup $N$ of $G$ for which the natural homomorphism $\mathcal{N}_{c_{1}, c_{2}, \ldots, c_{t}} M(G) \longrightarrow \mathcal{N}_{c_{1}, c_{2}, \ldots, c_{t}} M(G / N)$ is injective and hence the result follows by Theorem 2.4. Suppose $m=2$, then $G \cong \mathbb{Z} \oplus \mathbb{Z} \oplus \mathbb{Z}_{n_{1}} \oplus \ldots \oplus \mathbb{Z}_{n_{k}}$. Put $N$ to be the subgroup generated by $\left(0, n_{1}, \overline{0}, \ldots, \overline{0}\right)$ so $N \cong n_{1} \mathbb{Z}$ and $G / N \cong \mathbb{Z} \oplus \mathbb{Z}_{n_{1}} \oplus$ $\mathbb{Z}_{n_{1}} \oplus \ldots \oplus \mathbb{Z}_{n_{k}}$. Now by Theorem 2.7 we have $\left|\mathcal{N}_{c_{1}, c_{2}, \ldots, c_{t}} M(G)\right|=\left|\mathcal{N}_{c_{1}, c_{2}, \ldots, c_{t}} M(G / N)\right|$. Using Theorems 2.4 and Corollary 2.5 the result will follow. If $m=1$ then put $N=<$ $\left(n_{1}, \overline{0}, \ldots, \overline{0}\right)>$ and use a similar proof.

The reverse of the above lemma can be presented as follows.

Lemma 3.6. Let $G \cong \mathbb{Z}^{(m)} \oplus \mathbb{Z}_{n_{1}} \oplus \ldots \oplus \mathbb{Z}_{n_{k}}$ be an infinite finitely generated abelian group, where $n_{i+1} \mid n_{i}$ for all $1 \leq i \leq k-1$. If $m \geq 3$ then $G$ is $\mathcal{N}_{c_{1}, c_{2}, \ldots, c_{t}}$-capable.

Proof. Similar to Lemma 3.4 it is enough to show that there is no normal subgroup $N$ of $G$ for which $\mathcal{N}_{c_{1}, c_{2}, \ldots, c_{t}} M(G) \longrightarrow \mathcal{N}_{c_{1}, c_{2}, \ldots, c_{t}} M(G / N)$ is injective. Consider two cases (i) $N$ is infinite; and $(i i) N$ is finite.

In case $(i)$ trivially $r_{0}(G / N)<r_{0}(G)$, now by Theorem $2.7 r_{0}\left(\mathcal{N}_{c_{1}, c_{2}, \ldots, c_{t}} M(G / N)\right)<$ $r_{0}\left(\mathcal{N}_{c_{1}, c_{2}, \ldots, c_{t}} M(G)\right)$. So there is no injection between $\mathcal{N}_{c_{1}, c_{2}, \ldots, c_{t}} M(G)$ and $\mathcal{N}_{c_{1}, c_{2}, \ldots, c_{t}} M(G / N)$. In case $(i i) N \leq t(G)$ and hence $G / N \cong \mathbb{Z}^{(m)} \oplus \mathbb{Z}_{m_{1}} \oplus \ldots \oplus \mathbb{Z}_{m_{k}}$ where $m_{i+1} \mid m_{i}$ and $m_{i} \mid n_{i}$ for all $1 \leq i \leq k-1$, now by Theorem 2.7 we have $\mathcal{N}_{c_{1}, c_{2}, \ldots, c_{t}} M(G) \cong \mathbb{Z}^{\left(f_{m}\right)} \oplus \mathbb{Z}_{n_{1}}^{\left(f_{m+1}-f_{m}\right)} \oplus \ldots \oplus \mathbb{Z}_{n_{k}}^{\left(f_{m+k}-f_{m+k-1}\right)}$, and $\mathcal{N}_{c_{1}, c_{2}, \ldots, c_{t}} M(G / N) \cong$ $\mathbb{Z}^{\left(f_{m}\right)} \oplus \mathbb{Z}_{m_{1}}^{\left(f_{m+1}-f_{m}\right)} \oplus \ldots \oplus \mathbb{Z}_{m_{k}}^{\left(f_{m+k}-f_{m+k-1}\right)}$. Now by contrary assume that the natural homomorphism $\mathcal{N}_{c_{1}, c_{2}, \ldots, c_{t}} M(G) \longrightarrow \mathcal{N}_{c_{1}, c_{2}, \ldots, c_{t}} M(G / N)$ which will be called $f$ through the proof, is injective. It is easy to show that $t\left(\mathcal{N}_{c_{1}, c_{2}, \ldots, c_{t}} M(G)\right)=\mathbb{Z}_{n_{1}}^{\left(f_{m+1}-f_{m}\right)} \oplus \ldots \oplus$ $\mathbb{Z}_{n_{k}}^{\left(f_{m+k}-f_{m+k-1}\right)}$ and $t\left(\mathcal{N}_{c_{1}, c_{2}, \ldots, c_{t}} M(G / N)\right)=\mathbb{Z}_{m_{1}}^{\left(f_{m+1}-f_{m}\right)} \oplus \ldots \oplus \mathbb{Z}_{m_{k}}^{\left(f_{m+k}-f_{m+k-1}\right)}$. Also we have $f\left(t\left(\mathcal{N}_{c_{1}, c_{2}, \ldots, c_{t}} M(G)\right)\right) \subseteq t\left(\mathcal{N}_{c_{1}, c_{2}, \ldots, c_{t}} M(G / N)\right)$. As $f$ is injective we must have $m_{i}=n_{i}$ for all $1 \leq i \leq k$ so $t(G)=t(G / N)$ but $t(G / N)=t(G) / N$ and hence $N=0$ which completes the proof.

The latest lemmas classify all finitely generated abelian groups which are $\mathcal{N}_{c_{1}, c_{2}, \ldots, c_{t}}{ }^{-}$ capable, provided that $t \geq 2$ and $c_{1}=1$. Of the most famous examples of these varieties are $\mathcal{S}_{\ell}$ 's, the varieties of soluble groups of length at most $\ell$.

The case $c_{1} \geq 2$ or $t=1$ has different results but similar proof, so the proof are shortened. Examples of these varieties are $\mathcal{N}_{c}$ 's and $\mathcal{A}$, which are the varieties of nilpotent groups of class at most $c$ and abelian groups, respectively, for the case $t=1$, and polynilpotent varieties which are not solvable for the case $c_{1} \geq 2$. The following theorems are stated with the conditions $c_{1} \geq 2$ or $t=1$.

Theorem 3.7. Let $G \cong \mathbb{Z}_{n_{1}} \oplus \ldots \oplus \mathbb{Z}_{n_{k}}$ be a finite abelian group, where $n_{i+1} \mid n_{i}$ for all $1 \leq i \leq k-1$. Then $G$ is $\mathcal{N}_{c_{1}, c_{2}, \ldots, c_{t}}$-capable if and only if $k \geq 2$ and $n_{1}=n_{2}$. 
Proof. The proof is completely similar to the proofs of Lemmas 3.3 and 3.4. The only matter which may be considered is the fact that for an arbitrary finite abelian group $G \cong \mathbb{Z}_{n_{1}} \oplus \ldots \oplus \mathbb{Z}_{n_{k}}$ we have $\mathcal{N}_{c_{1}, c_{2}, \ldots, c_{t}} M(G) \cong \mathbb{Z}_{n_{2}}^{\left(f_{2}\right)} \oplus \mathbb{Z}_{n_{3}}^{\left(f_{3}-f_{2}\right)} \ldots \oplus \mathbb{Z}_{n_{k}}^{\left(f_{k}-f k-1\right)}$.

Theorem 3.8. Let $G \cong \mathbb{Z}^{(m)} \oplus \mathbb{Z}_{n_{1}} \oplus \ldots \oplus \mathbb{Z}_{n_{k}}$ be an infinite finitely generated abelian group, where $n_{i+1} \mid n_{i}$ for all $1 \leq i \leq k-1$ is $\mathcal{N}_{c_{1}, c_{2}, \ldots, c_{t}}$-capable if and only if $m \geq 2$.

Proof. The proof is similar to Lemmas 3.5 and 3.6. The only fact may be considered is the structure of the polynilpotent multipliers of $G$ and $G / N$ in the case $c_{1} \geq 2$ or $t=1$. More exactly the difference is the fact that we have $f_{2} \neq 0$, so the torsion free rank of $\mathcal{N}_{c_{1}, c_{2}, \ldots, c_{t}} M(G)$ is zero exactly when $G$ has the torsion free rank 1.

\section{Acknowledgments}

The authors are grateful to the referees for their careful works and suggestions that improved this paper.

The third author was in part supported by a grant from IPM (No. 83200037).

\section{References}

[1] R. Baer. Groups with preassigned central and central quotient group. Trans. Amer. Math. Soc., 1938, 44: 387-412.

[2] J. Burns, G. Ellis. On the nilpotent multipliers of a group. Math. Z., 1997, 226: 405-28.

[3] M. Hall, J. K. Senior. The Groups of Order $2^{n}(n \leq 6)$. MacMillan, New York, 1964.

[4] P. Hall. The classification of prime power groups. J. Reine Angew. Math., 1940, 182: 130141.

[5] B. Mashayekhy, M. Parvizi. Polynilpotent multipliers of finitely generated abelian groups. Int. J. Math. Game Theory Algebra, 2006, 16: 93-102.

[6] M. R. R. Moghaddam, S. Kayvanfar. A new notion derived from varieties of groups. Algebra Colloquium, 1997, 4: 1-11.

[7] M. Parvizi, B. Mashayekhy. An outer commutator multiplier and capability of finitely generated abelian groups. Comm. Algebra, 2010, 38: 588-600. 